\title{
Detecting and identifying response-compatible stimuli
}

\author{
JOCHEN MÜSSELER and BERNHARD HOMMEL \\ Max Planck Institute for Psychological Research, Munich, Germany
}

\begin{abstract}
Previous work indicates that action-control processes influence perceptual processes: The identification probability of a left- or right-pointing arrow is reduced when it appears during the execution of a compatible left-right-key press (Müsseler \& Hommel, in press). The present study addresses the question of whether this effect would also be observed in a detection task-that is, with judgments that do not require discriminating between left- and right-pointing arrows. Indeed, we found comparable effects in both the identification task and the detection task. This outcome is interpreted within a commoncoding framework, which holds that stimulus processing and action control operate on the same codes.
\end{abstract}

The present article deals with the relationship between perception and action and, in particular, with the effects of compatibility or incompatibility between stimuli and responses. Yet, in contrast to the numerous investigations on the impact of stimulus information on response selection (for overviews, see Hommel \& Prinz, 1997, and Proctor \& Reeve, 1990), our concern is with the reverse direction - that is, with the impact of action on perception. We found empirical evidence for such an impact in a recent study where subjects were presented with a masked left- or right-pointing arrow while executing a prepared left- or right-key press (Müsseler \& Hommel, in press). Surprisingly, when asked to identify the arrow at leisure afterwards, subjects were consistently better with incompatible relationships between response and arrow (e.g., left response, right-pointing arrow) than with compatible relationships (e.g., left response, left-pointing arrow).

Our interpretation of this finding rests on the assumption that stimulus processing and action control operate on the same kind of - and sometimes even identicalcodes (cf. the common-coding approach outlined by Prinz, 1992). According to this approach, a left response, for example, would be represented and controlled by the same cognitive LEFT code that is used in coding a left-pointing arrow (Müsseler \& Prinz, 1996). However, there is reason to assume that executing an action results in a selfinhibition of the action-related codes - hence, a refractory phase (MacKay, 1986). If so, executing a left response should be associated with a temporarily decreased sensitivity to LEFT stimuli, such as left-pointing arrows. The

This research was supported by Deutsche Forschungsgemeinschaft Grant Mu 1298/2-1 to the first author. The authors wish to thank Addie Ehrenstein for valuable comments, Birgitt ABfalg for carrying out the experiments, and Heidi John for stylistic suggestions. Correspondence should be addressed to either author at the Max Planck Institute for Psychological Research, Leopoldstr. 24, D-80802 Munich, Germany (e-mail: muesseler@mpipf-muenchen.mpg.de, or hommel@mpipf-muenchen. mpg.de). functional role of this sensitivity loss may be to protect a previously performed action from being activated again by the (response-compatible) stimuli produced by that action. Hence, executing an action may be associated with a kind of action-effect blindness (AEB; see Müsseler \& Hommel, in press), a transient insensitivity to stimuli sharing features or meaning with the action (i.e., to responsecompatible stimuli).

In the present three experiments, we explored in more detail what it means to be "blind" to a response-compatible stimulus. So far, AEB has been established in an identification task only, with judgments about the arrow direction. The present study addressed the question of whether the effect can also be observed in a detection task, with judgments that do not require the subject to discriminate between left- and right-pointing arrows.

At first sight, there are some reasons to doubt that AEB occurs with pure detection tasks. Logically, for a compatibility effect between a left-right response and a left- or right-pointing arrow to occur, arrow direction must be coded in some way. Yet, if detection is managed at processing stages preceding the identification stage or is based on less evidence than identification (e.g., Broadbent \& Broadbent, 1987; Manahilov, 1991), little or no direction information would be available in detection tasks; hence, there would be no AEB. However, the mere possibility of responding to a lesser amount of, or earlier available, sensory information does not preclude that identity-related information is also computed and, thus, may affect detection performance. So, detection and identification may differ more in degree than in type (e.g., Green, 1992; Thomas, 1985), which renders AEB in detection tasks at least possible.

In Experiment 1, we introduced the basic experimental design with an identification task, which - in an otherwise unchanged procedure-was replaced by a detection task in Experiment 2. Both experiments were designed to control for response-bias explanations. Finally, Experiment 3 tested a perceptual bias explanation. 


\section{EXPERIMENT 1}

We first attempted to replicate our previous finding that executing a response influences stimulus identification (Müsseler \& Hommel, in press). The procedure we used is shown in Figure 1: An arrow serving as response cue appeared on the screen $\left(S_{1}\right)$, and subjects were to prepare the corresponding response $\left(R_{1}\right)$ as long as they wished. Before emitting the prepared response, they performed an obligatory double-key press, which triggered the presentation of $\mathrm{S}_{2}$, the to-be-identified left- or right-pointing arrow (or a blank screen, see below). This way of triggering ensured that $S_{2}$ fell into the execution phase of $R_{1} . S_{2}$ was masked, and subjects were required to judge its identity (i.e., arrow direction) by pressing a left key or a right key at leisure. Our major concern was whether identification accuracy would depend on the compatibility between $R_{1}$, the left-right response, and the direction of $S_{2}$.

To control for guessing strategies, catch trials were introduced in which the mask appeared without an arrow. A problematic guessing strategy would be to systematically alternate keys from $R_{1}$ to $R_{2}$. This would favor correct judgments with $R_{1}-S_{2}$ incompatibility but incorrect judgments with compatibility, thus mimicking the hypothetical effect of AEB. A repetition bias would be less critical. It would favor compatible judgments over incompatible judgments and, thus, would work against, but not artificially produce, the expected effect.

\section{Method}

Twenty-one adults served as paid subjects in single, 1 -h sessions. All subjects reported having normal or corrected-to-normal vision. The experiments were carried out on a laboratory computer (Rhothron rho-prof 200) PC with black-on-white projection. The subjects placed their heads on a chin-and-forehead rest $50 \mathrm{~cm}$ in front of the monitor. Two microswitches, operated with the index and middle fingers of the right hand, served as response keys.

As illustrated in Figure 1 , the $S_{1}$ and $S_{2}$ arrows measured $0.6 \times 1.0^{\circ}$ The mask was a rectangle of $0.7^{\circ} \times 1.3^{\circ}$, with every pixel set with a probability of .66 in each trial. $S_{1}$ appeared for $504 \mathrm{msec}, 0.75^{\circ}$ below screen center, and pointed to the left or right with equal probability. Time to prepare $R_{1}$ and the preceding double-key press was unlimited, but the interval between them should be kept at a minimum (maximum = $1 \mathrm{sec}$ ). $\mathrm{S}_{2}$ appeared $0.75^{\circ}$ above screen center and was replaced by the mask after an individually adjusted presentation time (see below). $\mathrm{S}_{2}$ pointed to the left or right or was omitted with a probability of .33 each. The subjects judged $S_{2}$ direction by pressing the left or right key, which was released only after deletion of the mask, 1,008 msec after $R_{1}$ offset. The subjects were not informed about presentation probabilities or arrow omissions. They were asked to guess if uncertain. In case of incorrect responding $\left(R_{1}\right.$ or $R_{2}$ incorrect, or $R_{1}$ not within 1 sec after double-key press), they were presented with a beep and an error message.

The experimental phase consisted of sixteen 18-trial blocks $\left(2 \mathrm{R}_{1} \times\right.$ $3 \mathrm{~S}_{2} \times 3$ replications). It was preceded by a practice phase of eight $12-$ trial blocks ( $2 \mathrm{~S}_{2} \times 6$ replications), where $\mathrm{S}_{1}$ was omitted and, accordingly, no $R_{1}$ was required. $S_{2}$ presentation time was preset to $70 \mathrm{msec}$ and increased or decreased by $14 \mathrm{msec}$ when the error rate within a block was higher than $40 \%$ or lower than $10 \%$, respectively. Mean presentation time of the last three practice blocks was used in the experimental phase. However, when the error rates increased or decreased too much during the experiment, further adjustments were made according to the same criteria as in the practice phase.

\section{Results and Discussion}

On average, the double-key press was initiated $802 \mathrm{msec}$ after $S_{1}$ onset (initiation time; see Figure 1), $R_{1}$ was initiated $266 \mathrm{msec}$ later (interresponse time), and $R_{2}$ was made $863 \mathrm{msec}$ after mask offset (judgment time). As verified by planned comparisons, there was no indication that any one of these measures depended on the compatibility between $\mathrm{S}_{2}$ and $\mathrm{R}_{1}$ ( $p>.10$, or higher). The same was true for choice errors in $R_{1}$, which occurred in only

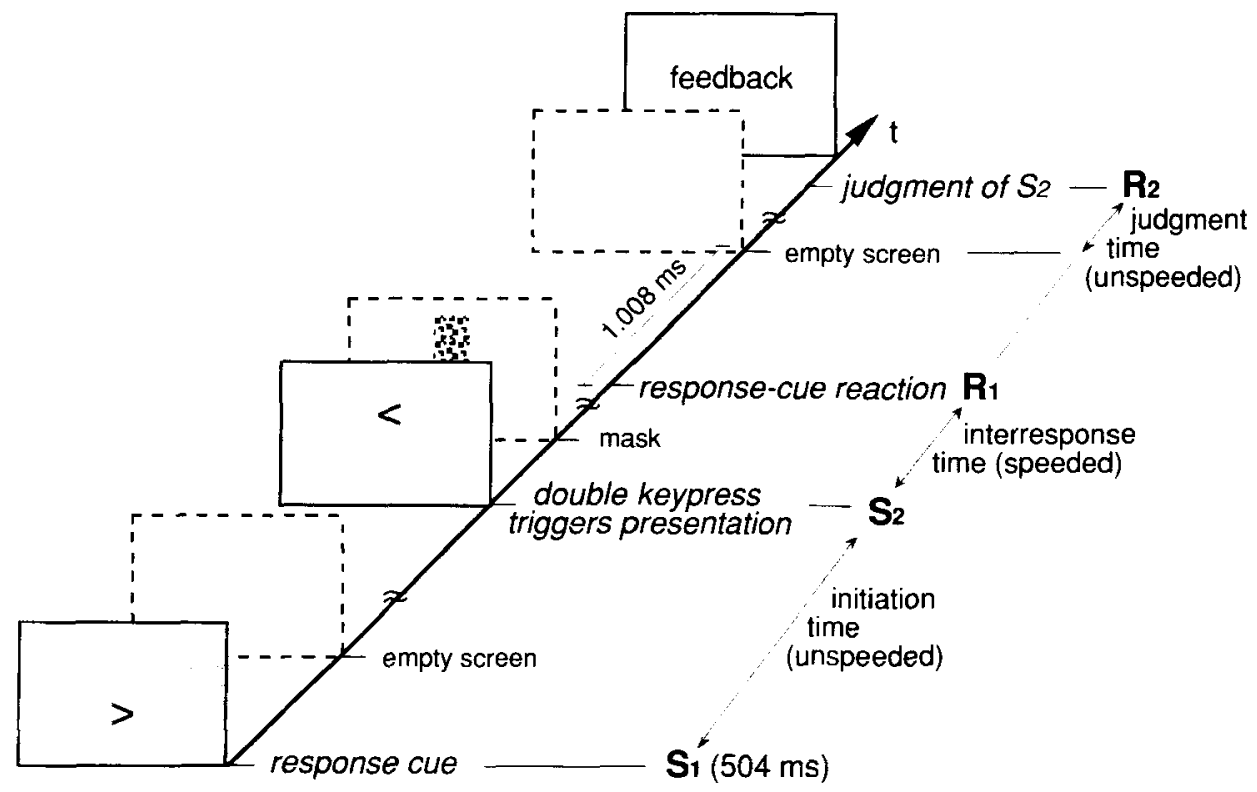

Figure 1. The series of events in the experiments: Subjects press both keys simultaneously before performing response $R_{1}$ cued by $S_{1}$ (here, the right key). While doing this, a masked stimulus $S_{2}$ is presented (an arrow or blank for an individually adjusted presentation time). The trial is completed with an unspeeded identification (Experiment 1) or a detection response (Experiments 2 and $3 ; \mathbf{R}_{2}$ ). 
$1.5 \%$ of the trials. These trials were excluded from the analyses of $\mathrm{S}_{2}$ identification rates.

On average, $S_{2}$ was presented for $36 \mathrm{msec}$. As shown in Figure 2, $S_{2}$ was less often identified correctly when $R_{1}$ and $S_{2}$ were compatible - that is, when the arrow's direction matched response side- than when they were incompatible (.626 vs. .706). In catch trials, there seemed to be a slight preference for "compatible" over "incompatible" guesses (.538 vs. .462; i.e., toward $R_{1}-R_{2}$ key repetition rather than alternation). An analysis of covariance was performed that removed the concomitant catchtrial variability from the arrow judgments. As expected, identification in compatible conditions again proved to be worse than that in incompatible conditions $[F(1,19)=$ $7.11, p=.015]$.

These results are clear-cut. First, the disadvantage in identifying response-compatible arrows, as compared with response-incompatible ones, nicely replicates the findings reported by Müsseler and Hommel (in press). Second, the tendency toward "compatible" guessing observed in the catch trials strongly suggests that, if anything, the disadvantage associated with compatible stimuli was underestimated, rather than overestimated, in the present results. In other words, the actual degree to which response-related processes make the system insensitive to response-compatible stimuli may be even larger than observed here.

\section{EXPERIMENT 2}

Experiment 2 was conducted to see whether or not AEB can also be found in a detection task. Besides providing a mean to test the (lower) limits of the effect, a detection task has at least one further advantage over identification tasks. With a task such as that used in Experiment 1, compatibility between $R_{1}$ and $S_{2}$ is always confounded with the compatibility relationship between $R_{1}$ and $R_{2}$. On the one hand, there is much evidence that this con-

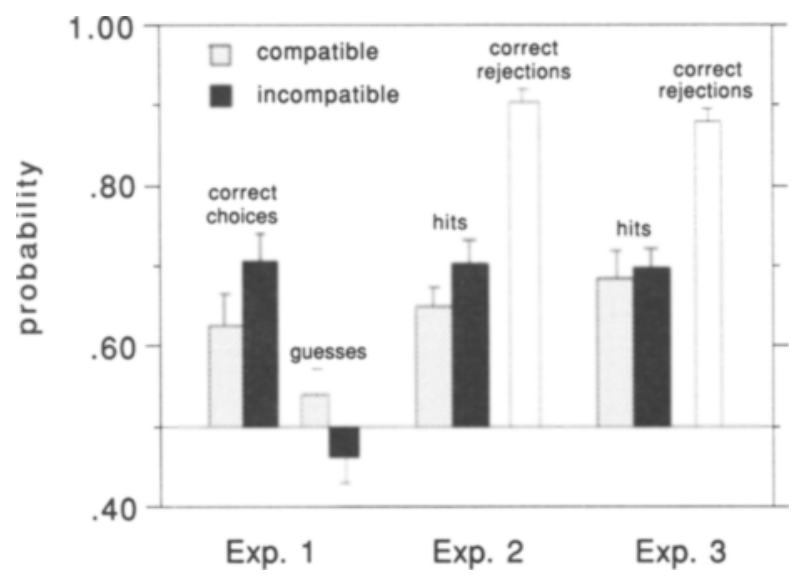

Figure 2. Mean probabilities and standard errors (between subjects) for correct choices and guesses in Experiment 1 and for hits and correct rejections in Experiments 2 and 3, as a function of compatibility between $R_{1}$ and $S_{2}$. Chance level is .5. founding is not responsible for the effect attributed to AEB. As evident from the blank trials in Experiment 1, response biases are not substantial and are of a direction that somewhat works against the effect. Moreover, Müsseler and Hommel (in press, Experiment 2) varied the similarity between $R_{1}$ and $R_{2}$ by replacing $R_{2}$ with verbal responses without any consequences for the effect. On the other hand, one may argue that some kind of relationship, albeit rather abstract, remains even in this case. Likewise, the argument from the blank trials may be countered by assuming that, for some reason, the critical response bias may show up without stimulus uncertainty only-hence, in nonblank trials-and, thus, cannot be estimated from blank-trial performance, in principle.

The most elegant way to counter these objections would be to avoid the critical confounding of $R_{1}-S_{2}$ and $R_{1}-R_{2}$ compatibility altogether. Fortunately, a detection task allows the decoupling of these two relationships by having subjects respond to the presence of a left- or right-pointing arrow by pressing the same key. Therefore, while the theoretically interesting compatibility between $R_{1}$ and $S_{2}$ was varied in Experiment 2 (as in Experiment 1), $R_{2}$ was held constant, at least as far as arrow-present trials were concerned. If, under these circumstances, AEB is still observed, a response-bias account of whatever kind could be easily rejected.

\section{Method}

Fifteen paid adults participated in the experiment. The only modification to Experiment 1 was that the subjects now were to detect $S_{2}$. For each subject, the experiment consisted of 384 trials ( 16 blocks $\times 24$ trials) in which a left arrow $(p=.25)$, a right arrow $(p=.25)$, or a blank $(p=.5)$ preceded the mask. The subjects were to judge the presence or absence of the $S_{2}$ by pressing a right or left key, respectively.

\section{Results and Discussion}

Initiation time, interresponse time, and judgment time were 837,265 , and $842 \mathrm{msec}$, respectively. None of these measures depended on the compatibility between $S_{2}$ and $\mathrm{R}_{1}(p>.10$, or higher $)$, nor did the $\mathrm{R}_{1}$ choice-error rates $\left(1 \%\right.$ overall). On average, the presentation time for $S_{2}$ was $23 \mathrm{msec}$, which was about two thirds of the time needed in Experiment 1. This suggests that less stimulus information was required to detect than to identify it, which comes as no surprise.

More importantly, response-compatible arrows were significantly less often detected than were responseincompatible arrows (.649 vs. $.704 ; t=3.09, p=.008$, see Figure 2). ${ }^{1}$ The false-alarm rate was $9.5 \%$. The effect of AEB demonstrated and replicated in Experiment 1 is not restricted to identification tasks but shows up in a detection task as well. True, the present effect was of a smaller size than that in Experiment 1, especially when one considers that the size of the latter was probably underestimated because of opposing guessing tendencies. Yet, in view of the fact that the $S_{2}$ presentation times were reduced, this is hardly surprising: If, in one trial or another, the mask terminated stimulus processing before the arrow's direction was determined, direction could not affect performance for trivial reasons. Nevertheless, Ex- 
periment 2 provided strong evidence for the fact that executing a prepared left-right response impairs the detection of an arrow pointing into the same direction.

\section{EXPERIMENT 3}

The results of Experiment 2 strongly suggest that we can rule out an account of AEB in terms of responseresponse relationships. Yet, what about the relationship between $S_{1}$, the response cue, and $S_{2}$ ? It is known from studies using rapid visual presentation techniques that people often fail to detect repetitions of visual stimuli (e.g., Kanwisher, 1987). Could this "repetition blindness" be the same phenomenon as we observed here? True, repetition blindness usually requires much shorter intervals between repetitions than present in our experiments. Nevertheless, $S_{1}$ and $S_{2}$ did represent a visual repetition with compatible conditions, so that the negative compatibility effect may have been due to the relationship between $S_{1}$ and $S_{2}$ rather than to that between $R_{1}$ and $S_{2}$.

In previous experiments (Müsseler \& Hommel, in press), we tested an explanation in terms of purely visual repetition by varying $S_{1}-S_{2}$ similarity or the compatibility between $S_{1}$ and $R_{1}$. The effect was not diminished when the written word left or right was used as $\mathrm{S}_{1}$ (and an arrow as $S_{2}$ ), and even when the instruction to $S_{1}$ was reversed-thus, it seems that $R_{1}$ impairs $S_{2}$ identification. However, all these experiments employed identification tasks; so, with detection tasks, a perceptual account may still apply. An obvious test of such an account is to omit $R_{1}$ in an otherwise unchanged task. While a stimulusrelated account would predict the same outcome as in Experiment 2, our own response-related account predicts that the effect disappears.

\section{Method}

Fifteen paid adults participated. The only modification to Experiment 2 was that the subjects were instructed to ignore the presentation of $S_{1}$. As a consequence, there was no speeded response $R_{1}$ after the double-key press.

\section{Results and Discussion}

Initiation time and judgment time were 960 and $496 \mathrm{msec}$, respectively. Neither one depended on $S_{1}-S_{2}$ compatibility ( $p>.05$, or higher). On average, the presentation time for $\mathrm{S}_{2}$ was $20 \mathrm{msec}$ (similar to that in Experiment 2). The false-alarm rate was $12.1 \%$. Yet, in contrast to the findings in Experiment 2, there was no indication whatsoever of an effect of $S_{1}-S_{2}$ compatibility on detection rates (.683 vs. .695 for compatible and incompatible conditions, respectively; $t=0.62, p>.25$; see Figure 2). Thus, the mere visual repetition or alternation of the stimuli $S_{1}$ and $S_{2}$ does not seem to be responsible for AEB.

However, it could be argued that the subjects did not "perceive" $S_{1}$ as in Experiment 2, by excluding it actively from further processing (e.g., by focusing another position) or by analyzing it only superficially. In this case, a vanishing of the effect would not be surprising. But note that, within one trial, the offset of $S_{1}$ serves as an indicator for the double-key press and that, therefore, $S_{1}$ cannot simply be overlooked. Moreover, although it might be true that a stimulus that requires a response is processed more extensively than is a stimulus that does not, it is well known from other paradigms (cf. the flanker paradigm) that mere presentation of a stimulus is sufficient to produce reliable, identity-related effects. Thus, there is reason to conclude from the present data that it is not the preceding stimulus presentation that matters for performance on $\mathrm{S}_{2}$, but the preceding response.

\section{GENERAL DISCUSSION}

The present three experiments provide further evidence for a novel effect that results from the coupling of an action with an action-related presentation: If a stimulus appears briefly before the overt onset of a prepared response (i.e., during the execution phase), identifying (Experiment 1) and detecting (Experiment 2) the stimulus is more difficult when its identity is related to the response. The demonstration of AEB in a detection task rules out possible response-bias explanations resting on the general assumption that the effect results from the relationship between the to-be-executed response $\left(R_{1}\right)$ and the response needed for indicating the stimulus presentation $\left(R_{2}\right)$. Since, in a detection task, this indication is independent of response -stimulus compatibility (i.e., the same response key is used for indicating compatible and incompatible presentations), this cannot be responsible for the effect.

The results of Experiment 3 rule out an explanation in terms of a simple perceptual bias: Obviously, the effect does not originate from the perceptual repetition that is given by the presentation of $S_{1}$ (needed to indicate the to-be-executed action) and the presentation of $S_{2}$ (to be detected). Otherwise, the mere presentation of $S_{1}$ without using it as a response indicator should also have produced an effect, but it did not. Further evidence against a critical role of $S_{1}-S_{2}$ similarity comes from our previous study (Müsseler \& Hommel, in press). In one experiment, we replaced $\mathrm{S}_{1}$ with the word left or right, and, in another experiment, subjects responded to the $S_{1}$ arrow in an incompatible way (i.e., "<" signaled a right-key press). Yet, the effect was not even diminished. So, although $A E B$ with a nonspatial $S_{1}$ still needs to be demonstrated, we are confident that such a demonstration is possible.

Altogether, these results strongly suggest that it is indeed the relationship between an action and an action-related stimulus that produces $A E B$. In the present study, this relationship was rather abstract: Responses differing in their horizontal location were paired with arrows pointing to the left or to the right; yet, the results indicate that even that abstract relationship can affect identification and detection substantially. In fact, according to the approach outlined by Müsseler and Hommel (in press), what matters is not the level at which the representations of response and stimulus overlap, but only that there is at least one feature they share, whether physically or semantically defined. If they do share such a feature -and thus are cognitively represented by partially overlapping codes - the overlapping part is involved in both executing the response and perceiving the stimulus (cf. the ideomotor notion of Greenwald, 1970). Under the assumption that after using a particular code for execution, this code enters a refractory period, stimulus perception should be impaired. In other words, executing a response should be accompanied by a temporary blindness to all of the stimuli with which the response shares features.

In this study, very similar results were obtained for the identification task and the detection task. This suggests that stimulus detection was not managed at a processing stage preceding identification; otherwise, direction information would not have been available, and no effect could have occurred. As it did, we must conclude that even if the task does not require the processing of identity information, this information is processed nevertheless. However, we would not go so far as to assume that detection never occurs without some degree of identification, 
as some authors (Green, 1992; Thomas, 1985) have proposed. Actually, there are many factors that are likely to affect the degree of identification present in detection tasks.

First, the more complex or the less controlled stimulus conditions in a particular experimental setting are, the more relevant identity information will be for discriminating the target stimulus from the surrounding or experimentally varied noise. For instance, identity information may not have been processed in our detection task if the only sensory information available in the situation had been provided by presentation of the target stimulus. Second, if identity information is processed automatically in each task, but would simply need more time to be computed than mere detection information, effects of identity would be the more likely, the less limited the presentation time is and the longer it takes to respond. Although, in our tasks, viewing time was limited by a mask, this is unlikely to terminate stimulus processing before any identity-related information is available, at least not in each trial. Moreover, if subjects are allowed to respond immediately to the presence of a stimulus, the response may often be carried out too quickly to be affected by identity information (Hommel, 1996). In our tasks, immediate responding was prevented; therefore, there was ample time for identity-related information to affect judgment. So, although the present findings are consistent with the view that lowering the task requirement to detection does not necessarily prevent identification of a stimulus, we doubt that this can be taken as a general rule.

\section{REFERENCES}

Broadbent, D. E., \& Broadbent, M. H. (1987). From detection to identification: Response to multiple targets in rapid serial visual presentation. Perception \& Psychophysics, 42, 105-113.

GREEN, M. (1992). Visual search: Detection, identification, and localization. Perception, 21, 765-777.

GREENWALD, A. G. (1970). Sensory feedback mechanisms in performance control: With special reference to the ideo-motor mechanism. Psychological Review, 77, 73-99

Hommel, B. (1996). S-R compatibility effects without response uncertainty. Quarterly Journal of Experimental Psychology, 49A, 546-571.
Hommel, B., \& Prinz, W. (1997). Theoretical issues in stimulusresponse compatibility. Amsterdam: Elsevier.

KanwISHER, N. G. (1987). Repetition blindness: Type recognition without token individuation. Cognition, 27, 117-143.

MaCKaY, D. G. (1986). Self-inhibition and the disruptive effects of internal and external feedback in skilled behavior. In H. Heuer \& C. Fromm (Eds.), Generation and modulation of action patterns (pp. 174-186). Berlin: Springer-Verlag.

MaNaHILOV, V. (1991). Grating detection and identification: Comparison of postadaptation reaction times. Vision Research, 31, 915-918.

Müsseler, J., \& Hommel, B. (in press). Blindness to responsecompatible stimuli. Journal of Experimental Psychology: Human Perception \& Performance.

Müsseler, J., \& Prinz, W. (1996). Action planning during the presentation of stimulus sequences: Effects of compatible and incompatible stimuli. Psychological Research, 59, 48-63.

Prinz, W. (1992). Why don't we perceive our brain states? European Journal of Cognitive Psychology, 4, 1-20.

Proctor, R. W., \& ReEve, T. G. (1990). Stimulus-response compatibility: An integrated perspective. Amsterdam: North-Holland.

ThомAs, J. P. (1985). Detection and identification: How are they related? Journal of the Optical Society of America A, 2, 1457-1467.

\section{NOTE}

1. To check whether the judgments $R_{2}$ are independent of $S_{2}$ direction and are not influenced by compatibility between $S_{2}$ and $R_{2}\left(S_{2}\right.$ present was indicated by a right-key press $R_{2}$ ), an additional analysis of variance was computed that included all four conditions (i.e., the two compatible $R_{1}-S_{2}$ combinations and the two incompatible ones). The only effect was produced by $\mathrm{R}_{1}-\mathrm{S}_{2}$ compatibility [.664 and $.634 \mathrm{vs.}$ .703 and $.705 ; F(1,14)=9.79, p=.007$; all other effects, $F\langle 1.00, p\rangle$ $.25]$, showing that the judgments were independent of $S_{2}$ direction.

(Manuscript received April 1, 1996; revision accepted for publication October 9, 1996.) 